\title{
Electron diffraction studies of supersonic jets. I. Apparatus and methods
}

\author{
Lawrence S. Bartell, Richard K. Heenan, and Makoto Nagashima \\ Department of Chemistry, University of Michigan, Ann Arbor, Michigan 48109 \\ (Received 9 September 1982; accepted 20 September 1982) \\ We describe the apparatus and methods used to obtain electron diffraction patterns of low-temperature \\ species produced from molecules seeded into supersonic expansions of helium or neon carrier gases. Although \\ systems designed for molecular beam or spectroscopic studies are unsuitable for electron diffraction, \\ alternative arrangements were found that give diffraction patterns of good quality. Characteristics of gas jets \\ issuing from different nozzle designs are discussed. Procedures are outlined for separating the desired signal \\ from considerable background scattering by the carrier gas and to correct for broad gas density profiles in \\ analyses of diffraction data.
}

\section{INTRODUCTION}

In recent years much attention has been given to spectroscopic studies of molecules cooled in supersonic expansions. ${ }^{1}$ Structural information about the cold species produced in such experiments is, however, often difficult to derive from spectroscopic data. Electron diffraction in appropriate cases is able to follow structural changes more directly. Problems that might be amenable to electron diffraction include low temperature conformational relaxation or the generation of van der Waals complexes or clusters. Of particular interest are systems which involve molecules seeded into various carrier gases. Because the requirements of electron diffraction are very different from those of molecular beam studies it was not altogether clear at the outset of this investigation whether it would be feasible to analyze the electron scattering from modest concentrations of molecules in a monatomic carrier gas. After an expansion has achieved the desired low temperature, the gas density is much less than that in conventional gas electron diffraction experiments. Research to be described in this and subsequent papers has confirmed that electron scattering can indeed yield valuable information about minor components in the presence of a carrier gas but, since the carrier contributes significantly to the scattering, the mole fraction of sample must be higher than in typical spectroscopic studies.

Electron diffraction requires the scattering gas to be quite well localized at some position along the electron beam and the background pressure to be low enough that molecules crossing the remainder of the electron path are few in number compared to those in the sample region. Therefore, in diffraction investigations, a skimmer between the gas nozzle and electron beam is essential, whereas in fluorescence spectroscopy, e.g., a skimmer is not normally required. On the other hand, since the electron beam would normally pass close to the skimmer exit, it is not necessary to have such a well collimated jet as is needed in typical molecular beam experiments and a single skimmer may suffice.

Extensive electron diffraction investigations of cluster formation in supersonic jets of various pure gases have been reported by workers in Orsay. ${ }^{2,3}$ In their work a double skimmer configuration was adopted to permit a high gas throughput with a relatively small pumping capacity. Several other diffraction studies of supersonic jets have been published, ${ }^{4,5}$ the principal interest to date centering on solid clusters of atoms or small molecules. The time seemed ripe to study more complex molecules seeded into rare gas carriers in view of the variety of relaxation and structural problems that could be envisaged. It was by no means clear whether the rapid cooling in microjets would be accompanied by enough collisions to induce the condensation products or conformational transformations that we sought. An investigation of different nozzle designs was therefore essential. Detection problems turned out to be eased, as expected, by the fractionation of heavier species towards the center of the jet. ${ }^{6}$

In the following sections features are described of an apparatus that has been successful in following both conformational relaxation and the formation of clusters with structures qualitatively different from those reported to date. Also outlined are procedures for analyzing diffraction data obtained under the atypical conditions of our experiments.

\section{EXPERIMENTAL}

\section{A. Apparatus}

The supersonic nozzle system which replaces the usual nozzle of our diffraction chamber ${ }^{7,8}$ is shown approximately to scale in Fig. 1. A conical skimmer separates the skimmer chamber from the diffraction chamber. This transmits only the central portion of the gas jet into the diffraction chamber where it intersects a $40 \mathrm{kV}$ electron beam about $2 \mathrm{~mm}$ beyond the skimmer exit. A brass tube of 3 in. internal diameter, bearing the skimmer and gas inlet pipe, runs through the wall of the diffraction chamber into the skimmer chamber where it is cut away to allow gas to escape to a cold trap above an oil diffusion pump. Since the nozzle is mounted 0.75 in. away from the axis of the brass tube, rotation of the tube translates the axis of the gas jet through the electron beam with little change in camera height. Screw mechanisms also allow the skimmer and nozzle to be independently moved towards or away from the electron beam. The inlet was designed so as to give as little obstruction as possible, consistent with con- 


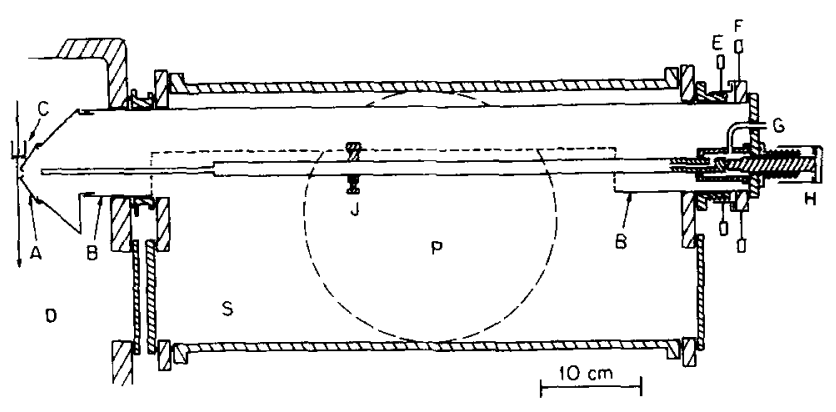

FIG. 1. A simplified cross sectional view of skimmer chamber $S$ connected to diffraction chamber $D$ by brass tube $B$ (diameter $3 \mathrm{in.}$ ). Interchangeable conical skimmer $A$ is mounted on the end of tube B. facing the gas nozzle. The center portion of tube $B$ is cut away (shown dashed) so that gas reflected from the skimmer may exit to a cold trap and oil diffusion pump (Varian VHS 10) in side arm P (diameter 10 in.). Gas mixture enters at $G$. Nozzle is centered on the skimmer aperture by screws J. Handles $\mathrm{E}$ change the skimmer-electron beam distance while screw $\mathrm{H}$ independently alters the nozzle-skimmer distance. Handles $F$ rotate tube $B$ to translate the gas jet laterally through the electron beam. Aperture C is a "cleanup" aperture through which the $40 \mathrm{kV}$ electron beam passes.

straints placed by the rest of the apparatus, to the flow of gas back around the nozzle into the skimmer chamber.

Strictly speaking the term "supersonic" can legitimately be applied to a conventional, noneffusive jet as used in gas diffraction. Commonly such jets flow from tubular nozzles $0.1-0.3 \mathrm{~mm}$ in diameter with sample pressures of a few Torr or more. While these jets experience substantial translational and rotational cooling, their constituent molecules undergo too few collisions before reaching the electron beam for the effects to be produced that we seek to study with the present apparatus. Our new system can cope with gas throughputs two orders of magnitude higher than those characteristic of our conventional diffraction experiments. Such throughputs lead to enormously enhanced nucleation and thermal effects. The skimmer chamber is evacuated by a Varian VHS10 oil diffusion pump (5000 $\mathrm{l} \mathrm{s}^{-1}$ for air, $7000 \ell^{-1}$ for He) backed by a Leybold Hereaus E150 mechanical pump $\left(44 \ell \mathrm{s}^{-1}\right)$. Experimental throughputs range up to 20 Torr $\ell \mathrm{s}^{-1}$ using helium or 10-12 Torr $\ell \mathrm{s}^{-1}$ with neon for short periods before the pumps are overloaded, at which point the pressure in the body of the skimmer chamber rises to $3 \times 10^{-3}$ Torr.

Geometric restrictions imposed by the bulk of the skimmer system preclude the carrying out of our standard alignment procedures. Positioning of the gas jet relative to the fixed electron optic axis is accomplished by translating the jet through the electron beam until the scattered electron current is maximized. Collecting plates to measure scattered current are mounted just above the rotating sector with wire brushes on the edge of the sector ball race to transfer the current. In initial experiments electrical noise of $\sim 10^{-11} \AA$ in the circuits was traced to the rubber drive wheel propelling the sector race. This friction-generated current was eliminated by replacing the rubber with an electrically conducting, graphite loaded, silicone elastomer. ${ }^{9}$
In addition to facilitating the alignment of the nozzle system, our provision for measuring the variation of scattered current as the axis of the gas jet is moved laterally serves another function. It allows us to deduce the density profile of the gas jet where it is probed by the electron beam. Such knowledge is essential to the derivation of precise amplitudes of vibration for sample molecules.

Crucial to the accurate measurement of diffraction patterns from low-density gas jets is the removal of unwanted background, or extraneous scattering. To this end we put a faster diffusion pump on the diffraction chamber (Varian VHS6, $2400 \mathrm{ls}^{-1}$ air, $3000 \mathrm{ls}^{-1} \mathrm{He}$ ) to reduce background gas, and we increased the height of our sector beam trap to $6 \mathrm{~cm}$, making it more efficient. These changes allowed us to increase exposure time an order of magnitude without accumulating an excessive extraneous background.

\section{B. Gas nozzles}

Three types of nozzle were investigated, namely, thin plate, tubular, and tapered glass. Thin plate apertures are circular holes of 25,50 , or $100 \mu \mathrm{m}$ diameter in steel disks $0.010 \mathrm{in}$. thick, supplied by Optimation Inc. Part of the mount for these is drawn to scale in the lower part of Fig. 2, which shows the disk pressed against a thin nylon or Teflon washer by a threaded steel cap. Tubular nozzles are standard stainless steel hypodermic needles cut off square to a length of around 5 $\mathrm{mm}$, with nominal inner diameters of 0.1 or $0.2 \mathrm{~mm}$. Tapered glass nozzles similar to those of Abraham et

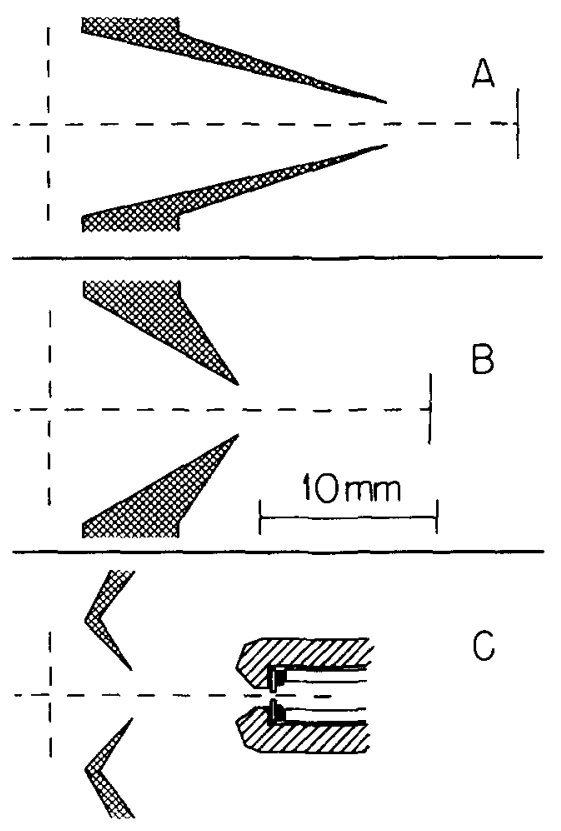

FIG. 2. Vertical sections of three conical skimmers drawn to scale, relating to curves $\mathrm{A}, \mathrm{B}$, and $\mathrm{C}$ of Fig. 3. The vertical dashed line marks the position of the $40 \mathrm{kV}$ electron beam $(\sim 2$ $\mathrm{mm}$ beyond the dkimmer exit). The axis of the gas jet is shown by a dashed line. The lower figure includes a section of the mount used for thin plate nozzles, a steel disk of thickness $0.010 \mathrm{in}$. with circular hole $(0.1 \mathrm{~mm}$ diameter), sealed by a Teflon washer under a threaded steel cap. 
al. ${ }^{10}$ were fabricated in a variety of sizes. They were seated in a Teflon collar in the inlet pipe and held in place by a threaded steel cap. Certain characteristics of the jets produced by these nozzles are described in a later section.

\section{Skimmers}

Several interchangeable, conical skimmers have been machined from brass to a sharp knife edge. Three that we shall compare here are illustrated to scale in Fig. 2. Scattered current profiles for these are shown in Fig. 3 using for each a mixture of 50 psia helium with $n$-pentane. First note that the profile for case $A$, the longest skimmer, is much wider than would be obtained by projecting straight lines from the nozzle exit through the skimmer entrance. This verifies that the flow was scattered appreciably by the skimmer. A second skimmer, case B, improved results somewhat, the illustrated peak scattered current only being lower than in case $A$ due to a longer nozzle to skimmer distance. In order to reduce geometric broadening of the gas jet we chose to make the skimmer entrance to electron beam distance as short as practicable for case $C$. Moving the nozzle closer to the skimmer increased the peak scattered current at the expense of increasing the background pressure in the diffraction chamber from $5 \times 10^{-5}$ Torr in case $B$ to $1 \times 10^{-4}$ Torr in case $C$.

The short skimmer $\mathrm{C}$ of Fig. 2, $2.8 \mathrm{~mm}$ long, was used in all subsequent work to be described here. We

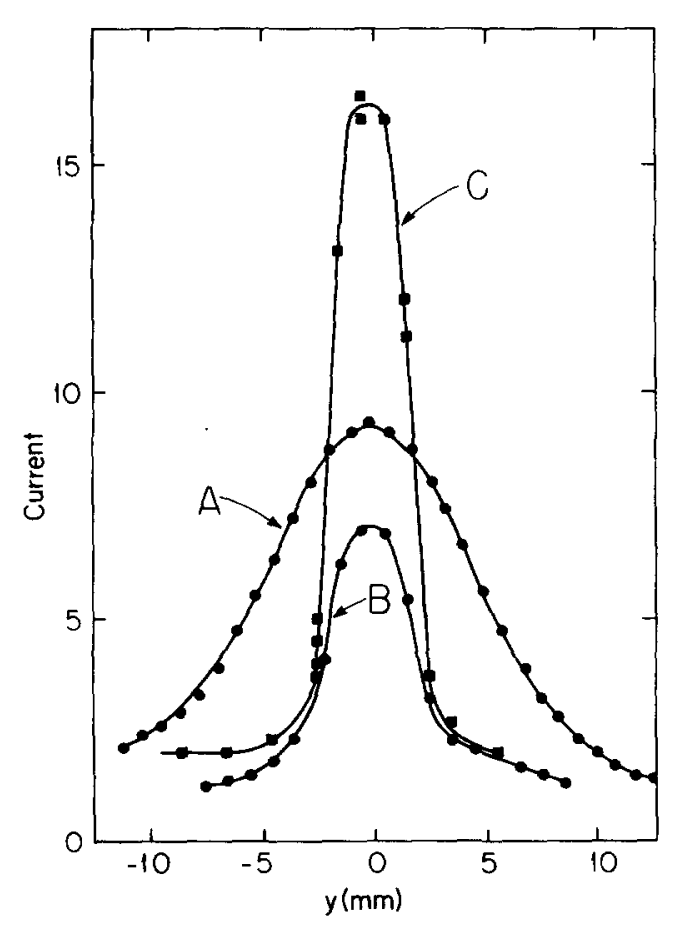

FIG. 3. Scattered current (arbitrary scale) against lateral displacement $y$ of gas jet for skimmer geometries A, B, and C of Fig. 2. In each case a thin plate nozzle of diameter $0.10 \mathrm{~mm}$ was used, with a total reservoir pressure of 50 psia helium containing room temperature $n$-pentane vapor ( 8.5 psia). Nozzle exit-to-skimmer entrance distances were, respectively, $7,11,8 \mathrm{~mm}$ for $\mathrm{A}, \mathrm{B}$, and $\mathrm{C}$.

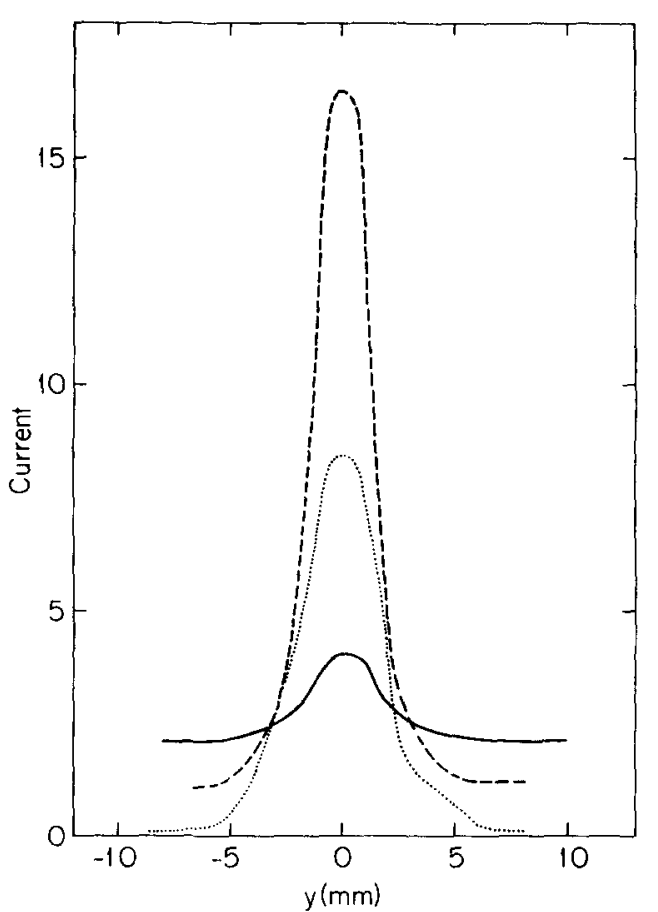

FIG. 4. Scattered current (on same scale as Fig. 3) against lateral displacement of gas jet using a tubular nozzle (length $5.6 \mathrm{~mm}$, diameter $0.12 \mathrm{~mm}$ ). The dashed line is for a total pressure of 70 psia helium containing 15 psia $n$-butane. Dotted curve is for 15 psia pure $n$-butane, solid curve for $40 \mathrm{psia}$ pure helium. In each case the nozzle exit-to-skimmer distance was $8 \mathrm{~mm}$ and skimmer $\mathrm{C}$ of Fig. 2 was used.

estimate the mean free path of gas jet molecules at the skimmer entrance to be around $0.1-1.0 \mathrm{~mm}$ with tubular or thin plate nozzles. Accordingly, the flow through the skimmer (diameter $2.66 \mathrm{~mm}$ ) is not properly effusive. Some interference of the flow must be expected which will tend to reheat the gas jet. Nevertheless, because the results we sought were achieved with skimmer $C$, no further research was carried out to optimize skimmer performance.

\section{Gas jet characteristics}

As discussed elsewhere ${ }^{11,12}$ it is possible to deconvolute scattered current profiles such as those illus trated in Fig. 3 to obtain gas density profiles. Due to problems that complicate the deconvolution, such as the poorly known radial fractionation of sample from carrier gas, we present here only the original current readings. A cylindrically symmetric, narrow, Gaussian gas density produces a current profile of exactly the same shape. A function appropriate for a simple unskimmed jet, "11 a "second order Witch of Agnesi" (the infinite order being a Gaussian) produces a current profile slightly less sharply peaked.

Effects of composition on gas flow, as monitored by electron scattering, are shown in Fig. 4 where the largest peak is for a mixture of helium plus $n$-butane at 70 psia through a tubular nozzle. The mixture, made by passing helium over liquid $n$-butane at $0^{\circ} \mathrm{C}$, contained a partial pressure of approximately 15 psia of 


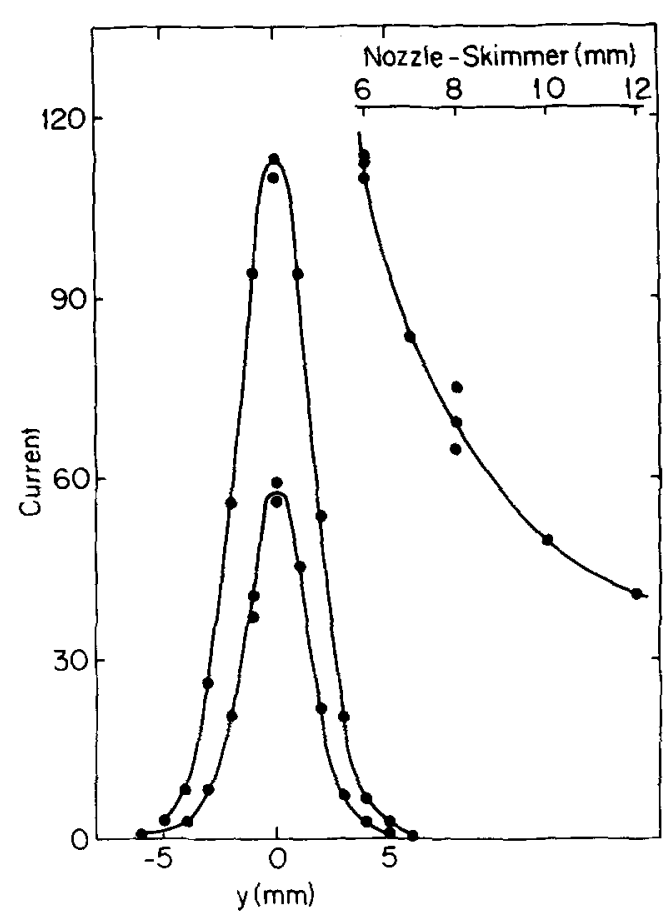

FIG. 5. Scattered current (on same scale as Figs. 3 and 4) against lateral displacement to gas jet using tapered glass nozzle 1 (entrance diameter $0.145 \mathrm{~mm}$, exit diameter $2.15 \mathrm{~mm}$, length $20 \mathrm{~mm}$ ). The lower curve is for pure $n$-butane (17 psia reservoir pressure) at a nozzle exit to skimmer distance of 8 $\mathrm{mm}_{\mathrm{i}}$ the upper curve is for $n$-butane (25 psia reservoir pressure) at a nozzle exit-to-skimmer distance of $6 \mathrm{~mm}$. The inset to the right shows the peak current, on the same scale, as the nozzle exit to skimmer distance was increased from 6 to $12 \mathrm{~mm}$ at 25 psia reservoir pressure.

butane. Most striking about the curves in Fig. 4 is that scattering from the mixture is twice as strong as from the same pressure of butane in a pure jet. Experimentally determined nozzle throughputs were 8.4 Torr $\ell \mathrm{s}^{-1}$ for the mixture, of which the butane fraction is 1.8 Torr $\ell \mathbf{s}^{-1}$, compared to 0.7 Torr $\ell \mathrm{s}^{-1}$ for pure butane at 15 psia. The increased butane throughput with helium carrier was primarily due to a decrease in mean molecular weight. However, acceleration of butane to higher velocities by helium tends to counteract the increase in throughput since butane molecules spend less time in the electron beam.

The third profile in Fig. 4, for pure helium at 40 psia, corresponding to a throughput of $6.8 \mathrm{Torr} \ell \mathrm{s}^{-1}$, suggests how the helium contribution to the mixture profile might look. With the pure helium however, background pressure in the diffraction chamber was much higher, as shown by the tails of the current profile and ion gauge readings. Since butane was very efficiently condensed onto a liquid nitrogen trap, the background pressure was due only to helium. Therefore it appears that less helium was passed through the skimmer in the case of the mixture than would have been expected from its nozzle throughput assuming a similar jet divergence. Conversely, diffusion of butane towards the center of the jet is apparent. The mass separation effect is well known $^{6}$ and, as described below, served to enhance cluster scattering.
Not yet examined is whether the scattered electron currents of Figs. 3 and 4 are adequate for electron diffraction exposures. In fact exposure times were in a reasonable range. As an example we consider the $\mathbf{7 0}$ psia mixture of Fig. 4 with, say, an electron beam current of $10^{-6} \AA$, a camera height of $21 \mathrm{~cm}$ and peak optical densities of 1.0 on the photographic plate. Suitable exposure times would then be $\sim 5 \mathrm{~s}$ with our $R^{2}$ sector or else $\sim 100 \mathrm{~s}$ with our $R^{3}$ sector. Times considerably shorter than these can be achieved by increasing the beam current or by altering the nozzle design as discussed below.

Changing to a tapered glass nozzle gave an increase of several fold in gas density at the electron beam. This is illustrated by the profiles for pure $n$-butane in Fig. 5 where the lower curve corresponds to conditions similar to those for the butane curve of Fig. 4. Remaining graphs in Fig. 5 show the effect of increasing the butane pressure and of varying the nozzle-to-skimmer distance. The peak current increases more slowly than the gas reservoir pressure as the gas distribution broadens, and the falloff with nozzle-skimmer distance is somewhat slower than inverse square, reckoning from the nozzle exit or somewhat faster, reckoning from the midpoint of the nozzle. Adding helium carrier to a pressure of 50 psia, with conditions otherwise the same as for the lower curve in Fig. 5, increased the peak scattered current by a further factor of 3 . The additional throughput swamped the diffraction chamber pumps after awhile, bringing the pressure above $10^{-3}$ Torr. Fortunately, short diffraction exposures were possible and these showed the presence of butane clusters.

Comparatively strong scattering also occurs with benzene or $\mathrm{SF}_{B}$ in helium or neon under conditions that produce clusters or microcrystallites. Trajectories of the heavy components from tapered nozzles are closer to the jet axis than divergent rays through the skimmer would imply. Indeed, with benzene or $\mathrm{SF}_{6}$ aggregates, flat disks of solid material only $6-11 \mathrm{~mm}$ in diameter collected on the cold trap surface some $100 \mathrm{~mm}$ downstream of the electron beam. A scattered current profile for an $S_{6}$ in helium gas jet is shown in Fig. 6 and a corresponding diffraction pattern in Fig. 7. Widths of the powder diffraction rings suggest, in this case, a typical crystal diameter on the order of $200 \AA$ and the lattice constant $[5.809(2) \AA]$ would correspond to a temperature in the bulk crystal of around $130 \mathrm{~K}{ }^{13}$ To obtain sufficient resolution the photographic plate was scanned at radius intervals of $0.03125 \mathrm{~mm}$, four times our usual sampling density. As has been mentioned by many previous author $s^{6,10,14}$ the advantages of tapered nozzles are a consequence of the greater number of collisions during expansion. They produce a better collimated jet and are more effective in producing clusters.

One phenomenon encountered was the cooling of the glass nozzle by expansions of benzene in helium (but not neon) to such an extent that benzene at its room temperature vapor pressure would condense and intermittently be sprayed by the helium flow into the vacuum chamber as macroscopic droplets. By introducing benzene at 


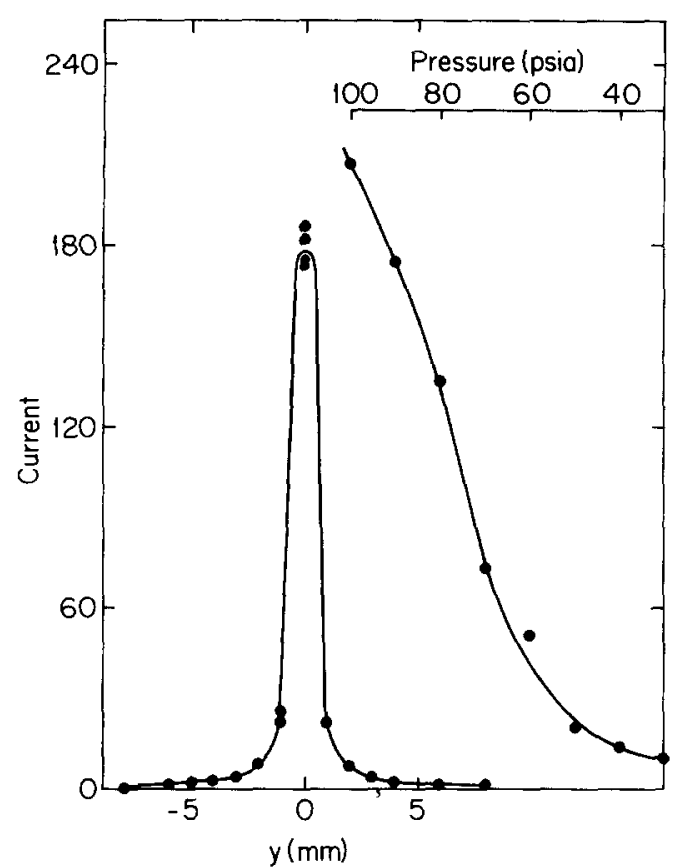

FIG. 6. Scattered current against lateral displacement of gas jet using tapered glass nozzle 6 (entrance diameter $0.128 \mathrm{~mm}$, exit diameter $1.9 \mathrm{~mm}$, length $30 \mathrm{~mm}$ ). The curve is for $12.5 \%$ $\mathrm{SF}_{6}$ in helium at a reservoir pressure of 90 psia. Diffraction patterns showed the presence of microcrystallites. Note the narrower gas jet compared to those of previous figures. The inset shows the peak current as the reservoir pressure was reduced from 100 to 30 psia.

lower pressure (from a $0^{\circ} \mathrm{C}$ ice bath) such condensation was avoided since, although the nozzle still cooled, it remained above the ice bath temperature.

Under the mild conditions of our expansions we have not seen any diffraction patterns from neon clusters which, if formed, might interfere with our seed molecule scattering. Observation of neon clusters by electron diffraction has recently been mentioned by Farges et al. ${ }^{2}$ Cluster structures so far examined appear to be independent of whether the carrier gas is helium or neon. We have yet to detect any scattering from rare gas van der Waals species.

\section{E. Microphotometer}

Our single beam microphotometer is based on a rugged 50 year old Sinclair-Smith frame and optical system with a filament lamp source supplied by a stabilized dc power supply. To cope with the increased number of plates that our latest studies have required, new detection circuits and computerized data collection have recently replaced an older card punch system.

Photographic plates are spun at four revolutions per second about the center of the diffraction pattern. Each plate is centered on the turntable by taking as a guide the shadow cast onto the emulsion by a fine wire fixed across the narrowest part of the sector opening. Optical components cast an image of a small region of the plate at approximately tenfold magnification onto a screen with a slit. Behind the slit is a dispersing lens and a photodiode (Cetron 1P39). The photodiode was found to give improved signal/noise ratio when the narrow pencil of incident light was defocused to spread over the full area of its collector plate. In order to ensure that electron counting statistics cannot introduce noise greater than about one part per ten thousand, the slit is opened far enough to make certain that the scanned area of the plate has received on the order of $10^{8}$ electrons for each data reading. Analog circuits amplify and integrate the diode current over periods of $0.25 \mathrm{~s}$ corresponding precisely to single revolutions of the photographic plate. Readings are initiated by the interuption of a light beam by a toothed ring on the precision screw drive which translates the rotating turntable. Data points are normally read at intervals of $0.125 \mathrm{~mm}$, a complete scan of 750 points across a plate diameter taking $4.25 \mathrm{~min}$. Timing pulses from a $60 \mathrm{~Hz}$ clock circuit also prompt readings of the integrator output by a Hewlett-Packard 3455A digital voltmeter. Voltages are transfered to a Tektronix 4051 computer where a program in BASIC immediately plots their values on a display screen. A least squares analysis of right- and left-hand readings determines the center of the trace and coefficients for baseline drift in the microphotometer circuits. The drift as shown by the difference of left- and right-hand voltages across the plate is usually less than $0.1 \%$ of the full scale reading. Noise in the readings has a height of around $0.04 \%$ of full scale.

A revised method to determine the conversion of optical absorbances into electron exposures has recently been described elsewhere. ${ }^{12}$ This reduces more effectively than previous procedures used in this laboratory the sensitivity of derived molecular parameters to variations in plate exposure time.

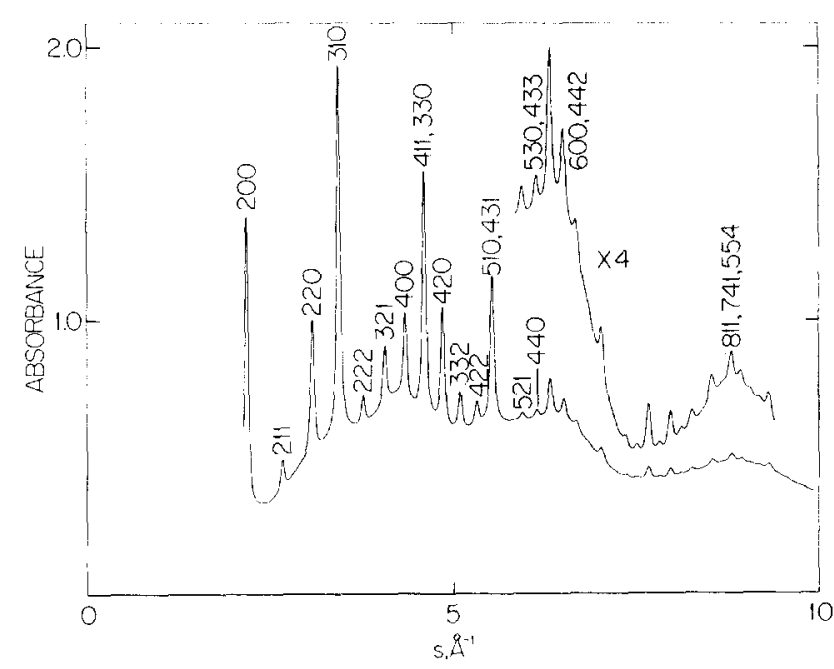

FIG. 7. Powder diffraction pattern of $\mathrm{SF}_{6}$ microcrystallites, superimposed on free $\mathrm{SF}_{6}$ and helium carrier scattering. Recorded in $0.4 \mathrm{~s}$ with an electron beam current of $0.23 \mu \mathrm{A}$. Reservoir pressure 90 psia of $12.5 \% \mathrm{SF}_{6}$ in helium, as for Fig. 6 . Absorbance on the photographic plate (emulsion blackness corrected) is shown against scattering variable $s$. Only the 110 reflection is inside the starting radius of cur $R^{2}$ rotating sector. All the observed structure may be indexed; only major rings are labeled here. 


\section{ANALYSIS OF DATA}

\section{A. Subtraction of carrier gas scattering}

The analysis of electron diffraction data at the University of Michigan traditionally proceeds through the reduced intensity function $M(s)$, the benefits of which have been discussed before. ${ }^{7}$ Before detailing the changes required to compensate for the scattering by the helium or neon carrier gas it will be helpful to outline the normal method.

Optical densities corrected ${ }^{12}$ to exposures $E(s)$ are divided by theoretical atomic scattering $I_{A}(s)$ to give a "leveled intensity" $I_{0}(s)$. In our structural least squares analysis we minimize the weighted sum of squared errors $\left[I_{0}^{\text {obs }}(s)-I_{0}^{\text {calc }}(s)\right]{ }^{2}$ The experimental leveled intensity is compared to the theoretical reduced intensity through an equation of the form

$$
R \cdot M(s)=\left[I_{0}(s) / B(s)\right]-1 \text {. }
$$

Index of resolution $R$ is ideally close to unity. Function $B(s)$ is a smooth background intended to compensate for arbitrary scaling of $I_{0}(s)$ and for any deficiencies in the theoretical background $I_{A}(s)$. Ideally $B(s)$ would have a constant value for all $s$, but in practice it is represented by a polynomial in $s$ together with an additional exponential term at low $s$. This provides a slowly varying function that may be adjusted in the least squares process.

Diffraction intensities of supersonic expansions often contain a considerable contribution $I^{c}(s)$ from monatomic carrier gas. The sample scattering may be written $I_{0}(s)=I_{0}^{\text {tot }}(s)-I_{0}^{c}(s)$, where both the total and carrier components have been leveled by the theoretical atomic scattering for sample gas only. Due to mass fractionation effects in the jet the proportion of carrier gas scattering is unpredictable even though the mole fraction of sample in the gas reservoir is known.

In some cases we have ignored the carrier gas contribution and have left the compensating background $B(s)$ to absorb its effect. If used routinely this would often result in an index of resolution $R$ much less than unity and might lead to erroneous values of amplitudes of vibration due to artificial changes in the rate of damping with $s$ of the sample scattering. For a quick preliminary analysis this approach is useful, particularly if the carrier gas component is small.

A better method is to subtract suitably scaled experimental carrier gas scattering from the total leveled intensity. A scale parameter $p$ was optimized in the general least squares analysis of

$$
I_{0}(s)=I_{0}^{\text {tot }}-p I_{0, \operatorname{expt}}^{c}(s) \text {. }
$$

Remaining deficiencies are still absorbed by the smooth background $B(s)$.

It is relevant to ask at this point the degree to which the carrier gas scattering obscures the desired molecular intensity function. As will be shown in later papers, we were encouraged by the fact that we have seen the same shapes of intensity functions for benzene or butane clusters over a range of carrier mole fractions and cluster concentrations, irrespective of whether helium or neon was used. As an example of our detec- tion capabilities we were able to see molecular scattering from 2 psia of benzene in 50 psia of neon from a tapered glass nozzle. In this case, unmistakably helped by mass separation, the molecular fraction of the total intensity was around one third of that for pure benzene. Since we believe that our averaged intensities contain significant information to a few parts in ten thousand the presence of carrier gas should not drastically impair our ability to discern molecular patterns in any of the cases considered here. Changes induced in the sample by collisions with the carrier should not therefore be masked by the carrier's own scattering.

\section{B. Corrections for broadened gas distributions}

As the scattered current profiles of Figs. 3-5 show, our gas jets are typically an order of magnitude broader than those of a conventional gas diffraction experiment. This results in blurring of patterns due to the distribution of camera heights $L$ of molecules deflecting electrons in different regions of the jet. Numerical integration of the normal theoretical intensity expressions over the gas distribution would give a good account of an experimental pattern. Fortunately, a simple approximation which requires less computation appears to be adequate. Note that a Gaussian probability distribution $\exp \left\{-\left(r-r_{a}\right)^{2} / 2 l^{2}\right\}$ in distance $r$ and $r_{a}$, introduces into the molecular intensity $M(s)$ a damping term $\exp \left\{-l^{2} s^{2} / 2\right\}$, where $l$ is a root-mean-square amplitude of vibration. A Gaussian distribution $\exp \left\{-\left(L-L_{0}\right)^{2} /\right.$ $\left.2 \sigma_{L}^{2}\right\}$ in the camera height $L$ has the same effect upon intensities recorded at the photographic plate as would a broader distribution in interatomic distances. If $\sigma_{L}$ $\ll L_{0}$, the apparent mean square amplitude $l_{L}^{2}$ will be related to the true mean square amplitude $l^{2}$ very nearly as

$$
l_{L}^{2}=l^{2}+\left(r_{a} \sigma_{L} / L\right)^{2} .
$$

Owing to our limited $s$ range, the failure of our jet density to follow exactly a Gaussian distribution will scarcely change results if we chose $\sigma_{L}$ realistically.

Values of $\sigma_{L}$ selected for least squares refinements of intensities may be estimated from scattered current profiles or else adjusted empirically to give the best fit when combined with calculated or expected $l$ 's. The effectiveness of this method was proven by an analysis of $n$-pentane pictures with jets deliberately made more diffuse than those of Figs. 4 and 5. For distributions such as in Fig. 4, with a nozzle-to-skimmer distance of $8 \mathrm{~mm}$ and a camera height of $214 \mathrm{~mm}$, a suitable value of $\left(\sigma_{L} / L\right)$ is 0.008 , though other similar values produce little change in the goodness of fit. As an example the bonded $\mathrm{C}-\mathrm{C}$ amplitude in $n$-butane might have a true value $l=0.0530 \AA$, whereas the corresponding uncorrected value is $l_{L}=0.0544 \AA$. Even for gas jets as broad as those in Figs. 4 and $5 \sigma_{L} / L$ is small compared to $l / r_{a}$, and little error is introduced into derived amplitudes of vibration by sample diffuseness once the system is characterized.

\section{CONCLUDING REMARKS}

We have reported here the methodology used for our electron diffraction studies of seeded supersonic jets, 
and have outlined some of the circumstances that make such experiments entirely feasible. Further details for particular molecular systems will be given in subsequent papers where we will concentrate upon the characterization of species generated in the jets.

\section{ACKNOWLEDGMENTS}

This research was supported by the National Science Foundation under grant numbers $\mathrm{CHE}-7682554$ and $\mathrm{CHE}-$ 7926480. Thanks are due to members of the chemistry and physics workshops of the University of Michigan, in particular to Mr. N. Johnston, Mr. W. Wolf, Mr. O. Steiner, Mr. R. Bell, and Mr. J. Babbit. Much assistance in experiments was provided by $\mathrm{Mr}$. M. Shetter.

${ }^{1}$ See, for example, W. Klemperer, J. Mol. Struct. 59, 161 (1980); A. Amirav, U. Even, and J. Jortner, Chem. Phys. 51, 31 (1980); R. E. Smalley, L. Wharton, and D. H. Levy, Acc. Chem. Res. 10, 139 (1977).

${ }^{2}$ J. Farges, M. F. de Feraudy, B. Raoult, and G. Torchet, Surf. Sci. Netherlands 106, 95 (1981).

${ }^{3} \mathrm{G}$. Torchet, thesis, University of Paris, Orsay, 1978; J. Farges, thesis, University of Paris, Orsay, 1977.

4A. Yokozeki, J. Chem. Phys. 68, 3766 (1978).
${ }^{5}$ S. S. Kim and G. D. Stein, Rev. Sci. Instrum. 53, 838 (1982); B. G. DeBoer and G. D. Stein, Surf. Sci. Netherlands 106, 84 (1981).

${ }^{6} \mathrm{~J}$. B. Anderson, in Molecular Beams and Low Density Gasdynamics, edited by P. Wegener (Dekker, New York, 1974); J. B. Anderson, Am. Inst. Chem. Eng. 13, 1188 (1967).

${ }^{7}$ L. S. Bartell, in Physical Methods of Chemistry, edited by A. Weissberger and B. W. Rossiter (Wiley-Interscience, New York, 1972), Vol. I, part IIId.

${ }^{8} \mathrm{R}$. L. Hilderbrandt, in Molecular Structure by Diffraction Methods, edited by G. A. Sim and L. E. Sutton, Specialist Periodical Report (Chemical Society, London, 1975), Vol. 3.

${ }^{9}$ We are grateful to Metex Corporation, New Jersey, for a sample of their CS-67 material.

${ }^{10}$ O. Abraham, J. H. Binn, B. G. DeBoer, and G. D. Stein, Phys. Fluids 24, 1017 (1981); O. Abraham, S. S. Kim, and G. D. Stein, J. Chem. Phys. 75, 402 (1981).

${ }^{11}$ L. S. Bartell, M. A. Kacner, and S. R. Goates, J. Chem. Phys. 75, 2730 (1981).

${ }^{12} \mathrm{~S}$. R. Goates, thesis, University of Michigan, 1981; S. R. Goates and L. S. Bartell, J. Chem. Phys. 77, 1886, 1877 (1982).

${ }^{13}$ E. J. Valente, R. K. Heenan, and L. S. Bartell (unpublished research).

${ }^{14} \mathrm{O}$. F. Hagena and H. von Wedel, Rarefied Gas Dymamics, Ninth Symposium, edited by M. Becker and M. Fiebig (DFVLR, Porz-Wahn, Germany, 1974); O. F. Hagena and W. Obert, J. Chem. Phys. 56, 1793 (1972). 